\title{
Cardiomiopatia Dilatada Reversível Relacionada a Hipertireoidismo
}

\begin{abstract}
RESUMO
A insuficiência cardíaca é uma das complicações mais conhecidas do hipertireoidismo, sendo mais comumente de alto débito, mas alguns pacientes evoluem com miocardiopatia dilatada e baixa fração de ejeção. Apresentamos paciente de 35 anos, sexo masculino, com hipertireoidismo, fibrilação atrial (FA) e insuficiência cardíaca grave com disfunção sistólica e fração de ejeção de $43 \%$. Após o tratamento definitivo do hipertireoidismo com radioiodo, houve reversão da insuficiência cardíaca, com melhora clínica e ecocardiográfica, incluindo normalização da fração de ejeção (69\%). Vários são os casos descritos de reversão da insuficiência cardíaca com o tratamento do hipertiroidismo, porém na sua maioria de insuficiência cardíaca de alto débito. Os mecanismos pelo qual o hipertireoidismo pode levar à disfunção sistólica, assim como seu tratamento, são discutidos. Concluímos que o tratamento do hipertireoidismo pode recuperar a miocardiopatia tireotóxica, mesmo em casos mais graves com comprometimento da função sistólica. (Arq Bras Endocrinol Metab 2007;51/9:1533-1538)
\end{abstract}

Descritores: Cardiomiopatia dilatada; Hipertireoidismo; Insuficiência cardíaca

\section{ABSTRACT}

\section{Reversible Dilated Cardiomyopathy Related to Hyperthyroidism.} Heart failure is one of the most known complications of hyperthyroidism, more commonly high-output heart failure, but some patients may develop dilated cardiomyopathy with low ejection fraction. We report a 35-year-old man, with hyperthyroidism, atrial fibrillation, and severe heart failure with $43 \%$ of ejection fraction. After the definitive treatment of the hyperthyroidism with radioiodine, heart failure was reverted, with symptomatic improvement and echocardiographic normalization including a normal ejection fraction (69\%). There are several cases of reversion of heart failure due to hyperthyroidism treatment, but most of them with a high-output heart failure. Mechanisms by which hyperthyroidism can lead to heart failure and its treatment are discussed. We conclude that treatment of hyperthyroidism may reverse this thyroid related heart failure, even in severe cases with systolic dysfunction. (Arq Bras Endocrinol Metab 2007;51/9:1533-1538)

Keywords: Dilated cardiomyopathy; Hyperthyroidism; Heart failure

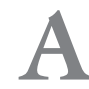

INSUFICIÊNCIA CARDÍACA É uma das complicações mais conhecidas do hipertiroidismo, sendo classicamente descrita como insuficiência cardíaca de alto débito em função das alterações hemodinâmicas e adrenérgicas que contribuem para o surgimento dessa condição (1). No entanto, alguns pacientes não somente iniciam um quadro de insuficiência apresentação de caso

\author{
LUIZ EDUARDO A. WILDEMBERG \\ LUCIANA LOPES DE SOUSA \\ LARA P. MONTEIRO DA FONSECA \\ Marcus V. LeItÃo de SOUZA
}

Serviço de Endocrinologia do Instituto Estadual de Diabetes e Endocrinologia Luis Capriglione (IEDE), Rio de Janeiro, RJ. 
cardíaca, mas ultrapassam a condição clássica da insuficiência cardíaca de alto débito, apresentando miocardiopatia dilatada e baixa fração de ejeção. Inúmeras são as condições que podem estar associadas a esta condição tão grave e por vezes irreversível em função do dano miocárdico já estabelecido.

Os autores relatam um caso de hipertiroidismo grave por doença de Graves (DG) associado à fibrilação atrial (FA) e insuficiência cardíaca com redução da fração de ejeção e posterior normalização da função miocárdica com o tratamento do hipertiroidismo. Serão discutidos os mecanismos que geram a insuficiência cardíaca no paciente hipertiróideo e a abordagem terapêutica, focalizando em especial o tratamento diferenciado a que devem ser submetidos tanto em relação à insuficiência cardíaca quanto à fibrilação atrial, já que constituem um grupo de pacientes normalmente mais jovens e sem outros fatores de risco.

\section{RELATO DO CASO}

CAC, masculino, 35 anos, iniciou quadro com sinais e sintomas típicos de hipertiroidismo, como insônia, nervosismo, taquicardia, hiperdefecação e aumento do volume cervical. Procurou atendimento médico, sendo indicada tireoidectomia, a qual não foi realizada devido à arritmia cardíaca diagnosticada por eletrocardiograma no pré-operatório. Permaneceu sem tratamento por aproximadamente um ano, tendo surgido no período edema de membros inferiores e dispnéia progressiva até a pequenos esforços. Negava dispnéia paroxística noturna ou ortopnéia.

Procurou emergência de um grande hospital devido a piora da taquicardia e palpitação, quando foi corroborado o diagnóstico de hipertiroidismo associado à fibrilação atrial. Nessa ocasião, foram iniciados propiltiouracil $1200 \mathrm{mg} /$ dia e propranolol 320 $\mathrm{mg} /$ dia, ambos por via oral. Referia ainda disfagia leve para sólidos, diplopia, proptose ocular e sensação de areia nos olhos. Após uma semana de tratamento na emergência sem melhora significativa foi transferido para o nosso serviço a fim de ajustar seu tratamento e programar tratamento definitivo do hipertiroidismo.

Ao exame físico na internação, apresentava-se levemente hipocorado, normotenso, taquicárdico (freqüência cardíaca de $120 \mathrm{bpm}$ ), com 74,5 kg, altura 1,80 m e índice de massa corporal de $22,9 \mathrm{~kg} / \mathrm{m}^{2}$, e com discreto tremor de extremidades.

A tireóide estava difusamente aumentada, com aproximadamente $100 \mathrm{~g}$, consistência fibroelástica, sem nódulos individualizáveis e sem sopro. Não foi palpada linfadenomegalia nas cadeias cervicais. $O$ exame ocular evidenciou edema palpebral bilateral mais pronunciado à esquerda, apenas com discreto distúrbio da convergência à esquerda.

Ao exame do sistema cardiovascular, foi observado ictus propulsivo, de duas polpas digitais, em sexto espaço intercostal esquerdo, ritmo cardíaco irregular, sem turgência jugular patológica. Apresentava hepatomegalia, com fígado palpável a aproximadamente $2 \mathrm{~cm}$ do rebordo costal direito, borda romba, sem outras alterações. Em membros inferiores apresentava edema bilateral com cacifo, sem sinais flogísticos e reflexo aquileu discretamente exacerbado.

Os exames complementares da admissão encontram-se na tabela 1 . O eletrocardiograma evidenciou uma fibrilação atrial de alta resposta, com freqüência ventricular de $110 \mathrm{bpm}$. O USG abdominal mostrou fígado de dimensões aumentadas $(16,5 \mathrm{~cm}$ na linha hemiclavicular), de textura sônica normal, sem dilatação das vias biliares intra e extra hepáticas.

No ecocardiograma transesofágico foi encontrado aumento das quatro cavidades cardíacas, disfunção global de VE moderada, com fração de ejeção de $43 \%$, discreto aumento no líquido fisiológico pericárdico, discreto derrame pleural esquerdo $<100 \mathrm{ml}$ e direito aproximadamente $50 \mathrm{ml}$.

Inicialmente foi trocado propiltiouracil (PTU) por metimazol (MMZ) $120 \mathrm{mg} /$ dia, com intuito de enviá-lo para dose terapêutica de iodo radioativo (DT) e podermos suspender o antitireoidiano em menor número de dias previamente à $\mathrm{DT}$ e minimizar o efeito radioprotetor do PTU como sugerido por Souza e cols. (2). Foram acrescentadas drogas para compensação da insuficiência cardíaca, como deslanósido e furosemida endovenosa, espironolactona, trocado o propranolol por carvedilol, além de ácido acetilsalicílico (AAS) $100 \mathrm{mg} /$ dia como profilaxia de tromboembolismo. Após estabilização da congestão pulmonar, o deslanósido foi suspenso, a furosemida foi trocada para via oral e foi iniciado aumento progres-

Tabela 1. Resultados de exames laboratoriais da admissão.

\begin{tabular}{lcc}
\hline & Resultado & $\begin{array}{c}\text { Valor de } \\
\text { referência }\end{array}$ \\
Tiroxina livre & $15,3 \mathrm{ng} / \mathrm{dL}$ & 0,8 a 1,8 \\
Triiodotironina total & $650,5 \mathrm{ng} / \mathrm{dL}$ & 60 a 181 \\
TSH & $<0,011 \mathrm{uU} / \mathrm{mL}$ & 0,35 a 5,5 \\
TRAb & $149 \mathrm{U} / \mathrm{L}$ & $<10$ \\
anti-TPO & $539 \mathrm{U} / \mathrm{mL}$ & $<35$ \\
TGO & $48 \mathrm{U} / \mathrm{L}$ & $<37$ \\
TGP & $71 \mathrm{U} / \mathrm{L}$ & $<41$ \\
Fosfatase alcalina & $173 \mathrm{U} / \mathrm{L}$ & 40 a 129 \\
$\gamma$ GT & $69 \mathrm{U} / \mathrm{L}$ & 11 a 49 \\
\hline
\end{tabular}


sivo do carvedilol de $3,25 \mathrm{mg} /$ dia até $25 \mathrm{mg} /$ dia. Concomitantemente, foi acrescentada dose baixa de captopril. Foi obtido bom controle clínico da insuficiência cardíaca.

Após controle da insuficiência cardíaca e do hipertiroidismo, foi indicada realização de DT para tratamento definitivo do hipertiroidismo. A cintigrafia da tireóide mostrou uma distribuição uniforme do radioiodo em ambos os lobos, com captação tiroidiana de $\mathrm{NaI}{ }^{131} 36 \%$ em 2 horas e $78 \%$ em 24 horas. Foi suspenso o MMZ cinco dias antes da DT e iniciado lítio na dose de $900 \mathrm{mg} /$ dia, sendo mantida esta dose até o $6^{\circ}$ dia pós-DT para evitar descompensação do hipertiroidismo, como sugerido por Bogazzi e cols. (3). Foram administrados $18 \mathrm{mCi}$ de ${ }^{131} \mathrm{I}$.

Paciente evoluiu com melhora progressiva tanto dos sintomas do hipertiroidismo quanto da insuficiência cardíaca após a administração da DT. A FA apresentou reversão no $3^{\circ}$ mês pós-DT, período em que estava clinicamente hipotiróideo, porém laboratorialmente ainda com TSH suprimido. Houve progressão para hipotiroidismo franco no $4^{\circ}$ mês após a DT, sendo iniciada reposição de levotiroxina. Algumas medicações para a insuficiência cardíaca foram sendo retiradas à medida que o paciente não apresentava evidência de insuficiência cardíaca. Aproximadamente um ano após a administração da dose terapêutica, foi realizado novo ecocardiograma, que evidenciou leve aumento do diâmetro diastólico final do VE, função contrátil global do VE preservada, fração de ejeção de $69,2 \%$, índices de função diastólica do VE normais, valvas cardíacas de aspecto morfológico normais, com regurgitação mitral leve.

\section{DISCUSSÃo}

O paciente em questão apresentava sinais e sintomas clássicos de hipertiroidismo por DG com duração superior a um ano, sem qualquer tratamento. Durante o curso de sua patologia tiroidiana, evoluiu com sinais claros de insuficiência cardíaca congestiva.

É sabido que o estado de hipertiroidismo leva a um aumento da demanda metabólica e, paralelamente a isso, ocorrem alterações cardiovasculares que são necessárias para atender a esse aumento da demanda metabólica. Por isso, a insuficiência cardíaca causada pelo hipertiroidismo é classificada como de alto débito, assim como ocorre em outras patologias como beri-beri, doença de Paget óssea e anemia grave. A relação do hipertiroidismo com o sistema cardiovascular sempre foi intensamente estudada. A própria fisiopatologia do hipertiroidismo explica parte das alterações cardiovasculares, pois o aumento do metabolismo basal imposto pelo excesso de hormônios tiroidianos circulantes leva a um aumento da volemia, redução da resistência vascular sistêmica e redução do tempo de circulação. Essas modificações no aparelho cardiovascular podem levar à hipertrofia ventricular esquerda, secundária ao aumento do trabalho cardíaco (4). Há incapacidade em aumentar a fração de ejeção com o exercício pela redução da reserva contrátil miocárdica e pela impossibilidade de incremento na vasodilatação, além de fadiga muscular. Existe, ainda, um maior potencial de arritmias atriais (as ventriculares são raras, pois a densidade de receptores $\beta$ adrenérgicos é maior no átrio), e a conjunção de todos esses fatores pode culminar com insuficiência cardíaca de alto débito $(4,5)$.

Entretanto, existem relatos de casos de insuficiência cardíaca de baixo débito, com redução da fração de ejeção, durante o estado de hipertiroidismo. O paciente em questão, durante o curso do hipertiroidismo, apresentou sinais clínicos de falência cardíaca, sendo realizado ecocardiograma que corroborou o diagnóstico clínico. A fisiopatologia dessa insuficiência cardíaca de baixo débito ainda não está estabelecida. Tem sido formulado que os hormônios tiroidianos podem alterar a expressão gênica de certas proteínas cardíacas, incluindo a cadeia pesada da miosina e a adenosina trifosfatase do retículo sarcoplasmático, o que leva à disfunção contrátil (5-7). Outro mecanismo proposto é a presença de taquicardia crônica no hipertiroidismo, levando à cardiomiopatia relacionada à taquicardia. É especulado que a taquicardia crônica causa depleção energética na célula miocárdica e anormalidade no alinhamento dos miócitos (8). Também foi demonstrado que a tireotoxicose impede o aumento da fração de ejeção ventricular esquerda durante o exercício (9). Atrofia cardíaca, semelhante à atrofia muscular esquelética causada pela alteração da composição protéica, também tem sido proposta como causa da cardiomiopatia dilatada na tireotoxicose. Em estudos de autópsia, dilatação ventricular esquerda e insuficiência cardíaca congestiva têm sido relatadas na ausência de outras formas de doença cardíaca. Fibrose intersticial e perivascular, hipertrofia miocárdica, necrose e edema celular também têm sido relatados (10).

A FA de alta reposta apresentada por este paciente provavelmente contribuiu para a deterioração do débito cardíaco. A contração atrial durante o ciclo cardíaco produz um enchimento adicional diastólico de VE em 30\%. Portanto, os átrios funcionam como bombas de ativação que aumentam a eficiência do bombeamento ventricular. Assim, a FA pode deteriorar a função cardíaca por perda da contração atrial 
com conseqüente redução do volume diastólico em $30 \%$ e pela redução do tempo de enchimento diastólico imposta pela freqüência cardíaca elevada. Assim, o controle da freqüência cardíaca deve ser perseguido de forma agressiva e concomitante ao controle do hipertiroidismo, mesmo na fase inicial do tratamento, enquanto ainda não foi revertida a congestão sistêmica, sendo esta uma das principais diferenças do tratamento da miocardiopatia tireotóxica da miocardiopatia dilatada convencional (11). Qual $\beta$-bloqueador deve ser utilizado é motivo de controvérsia, já que os estudos controlados que demonstraram diminuição de mortalidade na insuficiência cardíaca recomendam o uso de metoprolol, bisoprolol, carvedilol ou nebivolol $(12,13)$. Não existem estudos controlados avaliando especificamente pacientes hipertiróideos; no entanto, temos utilizado em nosso serviço o carvedilol nas doses recomendadas para insuficiência cardíaca, apesar de não haverem dados na literatura que contra-indiquem o uso ou mesmo a manutenção de $\beta$-bloqueadores não seletivos nesses pacientes (11). Na fase aguda, usamos rotineiramente digitálicos venosos para auxiliar o controle da freqüência cardíaca, notadamente enquanto mantémse a necessidade de diurético venoso. Caso haja necessidade de manutenção do digital, deve-se lembrar da necessidade de doses maiores em função do hipertiroidismo concomitante. Há controvérsias em relação à necessidade de anticoagulação de pacientes com FA secundárias a hipertiroidismo (14), mas dados sugerem que esses pacientes não apresentam risco de tromboembolismo maior que os com FA por outras causas, notadamente nos pacientes com menos de 55 anos de idade (15). Como se tratava de um paciente jovem, no qual foi realizado ecocardiograma transesofágico que não evidenciou trombos, foi optado por não iniciar anticoagulação com cumarínicos, sendo mantido apenas AAS em dose baixa. Normalmente, o AAS é visto com receio nos pacientes hipertiróideos descompensados, sendo classicamente contra-indicada para tratamento da febre em pacientes com crise tireotóxica por promover aumento da fração livre do T4. Mas isso ocorre em doses mais elevadas e a dose de $100 \mathrm{mg}$ é utilizada rotineiramente nesse grupo de pacientes em nosso serviço sem nenhuma intercorrência.

Em pacientes idosos com doença cardíaca, a sobrecarga cardíaca aumentada que resulta do hipertiroidismo pode agravar ainda mais a função cardíaca. O paciente em questão, entretanto, é jovem e não tem história prévia de cardiopatia. Assim, supomos que o aparecimento de insuficiência cardíaca de baixo débito deva ter relação com o tempo sem tratamento (mais de um ano) e com a gravidade do hipertiroidismo (T4 livre de $15 \mathrm{ng} / \mathrm{dl}$ ), porém não sendo esses os únicos mecanismos responsáveis pelas alterações nas proteínas contráteis e cardiomiopatia, principalmente em função de estudos sugerindo fatores genéticos relacionados ao surgimento de FA e eventualmente disfunção cardíaca (16).

Casos de reversão do quadro de insuficiência cardíaca decorrente do hipertiroidismo têm sido descritos. Umpierrez e cols. descreveram uma série de 7 pacientes com hipertiroidismo e insuficiência cardíaca, na qual houve normalização da fração de ejeção em 5 pacientes e evolução de disfunção severa para leve nos outros dois (18). Outros relatos semelhantes na literatura sugerem que, com a restauração do eutiroidismo, a disfunção cardiovascular pode ser normalizada (1926), uma vez que o fator desencadeante foi adequadamente tratado. Vale ressaltar que não são bem definidos os fatores que implicam maior ou menor chance de recuperação da função ventricular, assim como o tempo necessário para essa reversão varia de 1 semana após instituição do tratamento (21) até 24 semanas após estabelecimento do eutiroidismo (20). Um sumário das principais publicações referentes aos casos de hipertiroidismo com miocardiopatia dilatada reversível encontra-se na tabela 2. No caso em questão, hipertiroidismo foi tratado com droga antitireoidiana e o tratamento definitivo feito com o radioiodo. $\mathrm{O}$ paciente obteve melhora sintomática e ecocardiográfica do quadro de insuficiência cardíaca e evoluiu com hipotiroidismo quatro meses após a administração do iodo radioativo, em reposição com levotiroxina. O paciente encontra-se assintomático do ponto de vista cardíaco, estando, no presente momento, em uso apenas de dose mínima de inibidor da enzima de conversão, além da reposição de levotiroxina. O ecocardiograma revelou câmaras cardíacas de tamanho normais e função sistólica de VE preservada com fração de ejeção de $69 \%$. Uma comparação entre os ecocardiogramas antes e após o tratamento definitivo é encontrada na tabela 3.

Concluímos que, mesmo em casos graves de insuficiência cardíaca relacionados ao hipertiroidismo e com baixa fração de ejeção, o tratamento agressivo do hipertiroidismo e da insuficiência cardíaca pode recuperar a função miocárdica completamente, porém ainda não existem fatores preditores que definam quais pacientes permanecerão com disfunção ventricular e/ou miocardiopatia. Assim, sugerimos sempre a tentativa de redução progressiva e retirada das medicações normalmente utilizadas para tratamento da insuficiência cardíaca, após compensação clínica tanto do hipertiroidismo quanto da insuficiência cardíaca. 
Tabela 2. Resumo das publicações de miocardiopatia dilatada com disfunção sistólica em pacientes com hipertiroidismo.

\begin{tabular}{|c|c|c|}
\hline Referência & Características & Comentário \\
\hline Kantharia e cols. (19) & $\begin{array}{l}\text { Relato de } 1 \text { caso. } \\
\text { Sexo feminino, } 52 \text { anos }\end{array}$ & $\begin{array}{l}\text { Admissão em UTI com IC descompensada. } \\
\text { Normalizou FE } 6 \text { meses após eutiroidismo }\end{array}$ \\
\hline Watanabe e cols. (18) & $\begin{array}{l}\text { Relato de } 1 \text { caso. } \\
\text { Sexo masculino, } 28 \text { anos }\end{array}$ & $\begin{array}{l}\text { Admissão hospitalar com IC descompensada. } \\
\text { NYHA classe IV. } 2 \text { anos após tratamento, NYHA } \\
\text { classe I em uso de beta bloqueador, porém sem } \\
\text { reversão da FA. Sem descrição da FE }\end{array}$ \\
\hline Umpierrez e cols. (17) & $\begin{array}{l}\text { Relato de } 7 \text { casos. } \\
4 \text { sexo feminino e } 3 \text { masculino. } \\
\text { Média de } 47 \pm 4 \text { anos de idade }\end{array}$ & $\begin{array}{l}\text { FE média se elevou de } 28 \% \text { para } 55 \% .5 \text { pacientes } \\
\text { normalizaram completamente a } \mathrm{FE}\end{array}$ \\
\hline Jeyamalar e col. (21) & $\begin{array}{l}\text { Relato de } 1 \text { caso. } \\
\text { Sexo feminino, } 67 \text { anos }\end{array}$ & $\begin{array}{l}\text { Admissão hospitalar em edema pulmonar. FE de } 17 \% \text {. } \\
\text { Normalização da FE (72\%) após } 1 \text { ano do inicio do } \\
\text { tratamento }\end{array}$ \\
\hline Marti e cols. (22) & $\begin{array}{l}\text { Cintilografia miocárdica de } 13 \\
\text { pacientes com hipertiroidismo. } \\
2 \text { pacientes com disfunção sistólica }\end{array}$ & $\begin{array}{l}\text { Os } 2 \text { pacientes com disfunção sistólica apresentavam } \\
\text { alterações cintilográficas. A cintilografia e a disfunção } \\
\text { sistólica normalizaram }\end{array}$ \\
\hline Riaz e cols. (20) & $\begin{array}{l}\text { Relato de } 3 \text { casos. } \\
2 \text { pacientes sexo masculino } \\
\text { ( } 34 \text { e } 38 \text { anos) e } 1 \text { paciente sexo } \\
\text { feminino de } 51 \text { anos }\end{array}$ & $\begin{array}{l}3 \text { pacientes com reversão da disfunção sistólica, } \\
\text { sendo que o paciente mais jovem reverteu a disfunção } \\
\text { após } 1 \text { semana de tratamento }\end{array}$ \\
\hline Khandwala (10) & $\begin{array}{l}\text { Relato de } 1 \text { caso. } \\
\text { Sexo masculino, } 41 \text { anos }\end{array}$ & Normalização da FE após 1 mês de antitiroidiano \\
\hline Froeschl e cols. (23) & $\begin{array}{l}\text { Relato de } 1 \text { caso. } \\
\text { Sexo masculino, } 26 \text { anos }\end{array}$ & $\begin{array}{l}\text { Admissão em UTI por IC descompensada. Baixa FE } \\
\text { revertida após } 1 \text { mês de antitiroidiano. Óbito com disfunção } \\
\text { de múltiplos órgãos após complicações infecciosas na UTI }\end{array}$ \\
\hline Ngo e col. (24) & $\begin{array}{l}\text { Relato de } 1 \text { caso. } \\
\text { Sexo masculino, } 32 \text { anos }\end{array}$ & $\begin{array}{l}\text { Admissão em UTI, IC descompensada e FE } 25 \% \text {. Alta após } \\
9 \text { dias de internação. Sem novo ecocardiograma }\end{array}$ \\
\hline Siu e cols. (25) & $\begin{array}{l}\text { Análise prospectiva de } 591 \\
\text { pacientes com hipertiroidismo. } \\
34 \text { pacientes com IC }\end{array}$ & $\begin{array}{l}\text { 16/34 com IC tinham disfunção sistólica.12/16 reverteram a } \\
\text { disfunção sistólica ( } 75 \%)\end{array}$ \\
\hline
\end{tabular}

IC: Insuficiência cardíaca, FE: fração de ejeção do ventrículo esquerdo, UTI: unidade de tratamento intensivo, NYHA: classificação funcional da insuficiência cardíaca da New York Heart Association, que vai de I-IV, onde IV representa a descompensação máxima.

Tabela 3. Comparação entre ecocardiogramas pré- e póstratamento definitivo.

\begin{tabular}{lccc}
\hline & $\mathbf{0 6 / 0 4 / 0 5}$ & $\mathbf{2 2 / 0 4 / 0 6}$ & $\begin{array}{c}\text { Valores de } \\
\text { referência }\end{array}$ \\
VE sístole & $5,2 \mathrm{~cm}$ & $3,8 \mathrm{~cm}$ & Até $3,9 \mathrm{~cm}$ \\
VE diástole & $6,8 \mathrm{~cm}$ & $6,3 \mathrm{~cm}$ & Até $5,6 \mathrm{~cm}$ \\
$\mathrm{VD}$ & $2,8 \mathrm{~cm}$ & $2,0 \mathrm{~cm}$ & Até $2,6 \mathrm{~cm}$ \\
$\mathrm{AE}$ & $4,5 \mathrm{~cm}$ & $4,0 \mathrm{~cm}$ & Até $4,0 \mathrm{~cm}$ \\
$\mathrm{FE}$ & $43 \%$ & $69,2 \%$ & $>53 \%$ \\
\hline VE: &
\end{tabular}

VE: ventrículo esquerdo, VD: ventrículo direito, $\mathrm{AE}$ : átrio esquerdo, FE: fração de ejeção do ventrículo esquerdo

\section{REFERÊNCIAS}

1. Klein I, Ojamaa K. Thyroid hormone and the cardiovascular system: from theory to practice. J Clin Endocrinol Metab 1994;78:1026-7.

2. Souza MVL, Buescu A, Vaisman M, Souza HF, Luiz RR. Efeito do propiltiouracil sobre a eficácia da dose terapêutica de iodo radioativo (i-131) no hipertiroidismo por doença de Graves. Arq Bras Endocrinol Metab 2006;50:1088-95.

3. Bogazzi F, Bartalena L, Campomori A, Brogioni S, Traino C, Martino $F$, et al. Treatment with lithium prevents serum thyroid hormone increase after thionamide withdrawal and radioiodine therapy in patients with Grave's disease. $\mathbf{J}$ Clin Endocrinol Metab 2002;87:4490-5.

4. Woebker KA. Thyrotoxicosis and the heart. N Eng J Med 1992;327:94-8.
5. Polikar P, Burger AG, Scherrer U, Nicok P. The thyroid and the heart. Circulation 1993;87:1435-41.

6. Klein I, Ojamaa K. Thyroid hormone and the cardiovascular system. N Eng J Med 2001;344:501-9.

7. Ojamaa K, Klemperer JD, MacGilvray SS, Klein I, Samarel A. Thyroid hormone and hemodynamic regulation of $\beta$-myosin heavy chain promoter in the heart. Endocrinology 1996;137:802-8

8. Cruz FE, Cheriex EC, Smeets JL. Reversibility of tachycardiainduced cardiomyopathy after cure of incessant supraventricular tachycardia. J Am Coll Cardiol 1990;16:739-44.

9. Kahaly GJ, Wagner S, Nieswandt J, Mohr-kahaly S, Ryan TJ. Stress echocardiography in hyperthyroidism. J Clin Endocrinol Metab 1999;84:2308-13.

10. Khandwala HM. A case of congestive heart failure due to reversible dilated cardiomyopathy caused by hyperthyroidism. South Med J 2004;97:1001-3.

11. Klein IL, Danzi S. The cardiovascular system in thyrotoxicosis. In: Braverman LE, Utiger RD (eds). Werner and Ingbar's The Thyroid: A Fundamental and Clinical Text. $9^{\text {th }}$ ed. Philadelphia: Lippincott Willians \& Wilkins, 2005. pp. 559-68.

12. Jessup M, Brozena S. Heart failure. N Engl J Med 2003;348:2007-18.

13. Stoschitzky K, Stoschitzky G, Brussee H, Bonelli C, Dobniq H. Comparing beta-blocking effects of bisoprolol, carvedilol and nebivolol. Cardiology 2006;106:199-206.

14. Fuster V, Rydén LE, Crijns HJ, Ellenbogen KA, Kay GN, Prystowsky EN, et al. ACC/AHA/ESC 2006 guidelines for the management of patients with atrial fibrillation. J Am Coll Cardiol 2006;48:149-246.

15. Petersen $P$, Hansen JM. Stroke in thyrotoxicosis with atrial fibrillation. Stroke 1988;19:15-8. 
16. Schreieck J, Dostal S, von Beckerath N, Wacker A, Flory M, Weyerbrock S, et al. C825T polymorphism of the G-protein $\beta_{3}$ subunit gene and atrial fibrillation: Association of the TT genotype with reduced risk for atrial fibrillation. Am Heart $J$ 2004; 148:545-50.

17. Umpierrez GE, Challapalli S, Patterson C. Congestive heart failure due to reversible cardiomyopathy in patients with hyperthyroidism. Am J Med Science 1995;310:99-102.

18. Watanabe E, Ohsawa H, Noike H, Okamoto K, Tokuyama A, Kanai $M$, et al. Dilated cardiomyopathy associated with hyperthyroidism. Int Med 1995;34:762-7.

19. Kantharia BK, Richards HB, Battaglia J. Reversible dilated cardiomyopathy: an unusual case of thyrotoxicosis. Am Heart J 1995; 129:1030-2.

20. Riaz K, Forker AD, Isley WL, Hamburg MS, McCullough PA. Hyperthyroidism: curable cause of congestive heart failure. Congest Heart Fail 2003;9:40-6.

21. Jeyalamar R, Chan SP. A reversible dilated cardiomyopathy due to thyrotoxicosis. Int $\mathbf{J}$ Cardiol 1995;52:83-4

22. Marti V, Ballester M, Rigla M, Narula J, Bernà L, Pons-Lladó $\mathrm{G}$, et al. Myocardial damage does not occur in untreated hyperthyroidism unless associated with congestive heart failure. Am Heart J 1997;134:1133-7.
23. Froeschl M, Haddad H, Commons AS, Veinot JP. Thyrotoxicosis - an uncommon cause of heart failure. Cardiovasc Pathol 2005; 14:24-7.

24. Ngo AS, Lung Tan DC. Thyrotoxic heart disease. Resuscitation 2006;70:287-90.

25. Siu CH, Yeung CY, Lau CP, Kung AW, Tse HF. Incidence, clinical characteristics and outcome of congestive heart failure as the initial presentation in patients with primary hyperthyroidism. Heart 2007;93:483-7.

\section{Endereço para correspondência:}

Luiz Eduardo Armondi Wildemberg

Rua Lauro Muller 96/803

22290-160 Rio de Janeiro, RJ

E-mail: Iwildemberg@hotmail.com 